\title{
Can Artificial Intelligence Technology Replace Human Scribes?
}

\author{
Madison Anzelc, BS, BA; Craig G. Burkhart, MD; Craig N. Burkhart, MD
}

7 he personal connection between patients and physicians has evolved over the last decade with advances in medicine, technology, and the overwhelming impact of electronic medical records (EMRs). The average primary care physician spends 5.9 hours of their 11.4-hour workday doing various tasks in the EMR. ${ }^{1}$ With approximately half of a physician's workday dedicated to writing patient notes, billing, and managing their inbox, the other half of the day needs to be sparingly allotted across their total patient load.

This progression of increased EMR time demands and reduced time interacting with patients has led to the development of various advantageous strategies to minimize the physician's workload and shift the focus back to the patient. Two paramount examples that can maximize the physician's time and the patient's individualized care are the use of medical scribes as well as technology to write notes and accomplish various office tasks. Both reduce the physician's workload and allow for more patient-focused interactions but via different methods. When considering which practice to employ, a physician must weigh the positive and negative aspects of both modalities, particularly dermatologists who utilize these options to streamline high patient loads.

\section{Medical Scribes in Dermatology}

A scribe is defined as a staff member who records patientphysician interactions in real time and functions as the "physician's partner in the clinical encounter." ${ }^{2}$ A variety of staff members can serve as scribes, such as medical assistants and registered nurses (RNs), but the majority of scribes are prehealth students (eg, premedical, prenursing, pre-physician assistant). ${ }^{3}$ In this modality of patient information recording, the physician brings the scribe into the examination room and introduces them to the patient, and the scribe proceeds to record the encounter directly into the EMR. After the encounter, the physician then is able to review the completed notes and make the necessary changes before finalized submission. This process drastically reduces the physician's workload and also may have a lasting impact on the scribe. Aside from financial compensation, scribes also are offered a very in-depth clinical experience. Especially for prehealth students, scribing can be an eye-opening phase of their progression toward a future career in medicine. These students are able to immerse themselves in the clinical setting and truly experience the medical field through active participation in patient care. Robert et $\mathrm{al}^{2}$ commented on the professional development of prehealth students through scribing and self-reflection on their clinical experiences involving human suffering, empathy, power dynamics, and social inequality. Scribing allows prehealth students to begin to develop the critical skills necessary to succeed in the medical field at an earlier stage of their career development through real-time clinical engagement. This can be a motivational learning experience and can help these students to become more empathetic, understanding, and well-rounded providers in their future careers.

It is important to consider that human scribes currently are the status quo. They have been used reliably in the clinical setting for more than a decade, and it has been proven that their use is advantageous for physicians. Overall, the increased productivity and long-term effects of the immersive experiences that scribes encounter on a daily basis are important considerations when physicians decide to seek assistance in reducing their workload.

Ms. Anzelc and Dr. C.G. Burkhart are from the University of Toledo College of Medicine and Life Sciences, Ohio. Dr. C.G. Burkhart also is from the Ohio University Heritage College of Osteopathic Medicine, Athens. Dr. C.N. Burkhart is in private practice, Cary, North Carolina. The authors report no conflict of interest.

Correspondence: Madison Anzelc, BS, BA, 745 Washington St, Unit 704, Toledo, OH 43604 (Madison.anzelc@rockets.utoledo.edu). doi:10.12788/cutis.0402 


\section{Virtual Technology and Artificial Intelligence in Dermatology}

Another way to reduce the physician's daily workload is through virtual technology and artificial intelligence (AI)-based programs. There have been many varieties of technology developed over the last decade to coincide with the rising EMR work requirements. Virtual technology allows for a wide variety of utilization in the medical clinic that can vary from virtual assistants who record patient encounters, such as Hello Rache (Temark International, Inc), to audio programs such as DeepScribe (DeepScribe Inc) that listen to the patient-physician interaction and utilize an AI-based machine to concurrently convert the audio to written documentation in the EMR.

Among the available options, the most similar to the scribe method seems to be programs such as Hello Rache that provide a virtual assistant-often an $\mathrm{RN}-$ who can assist in completing a multitude of tasks, such as referrals, telephone calls, transcription of dictation, and other office needs. Similar to scribing, the virtual assistant can be brought into the room to chart the notes from the visit in real time into the EMR. Although this seems similar to conventional scribing, there are 3 glaring differences in the virtual approach. The first is that the use of a tablet, computer, or other technology source is required to bring the virtual assistant in the room to listen and observe the patient interaction. This increases ease of use and allows the physician to move seamlessly between patient encounters. However, the utilization of technology also adds a layer of potential problems to the physician's workflow, such as unreliable Internet connection, the need for battery power, and data storage requirements. The second major difference is the fact that the virtual assistant recording the notes into the EMR is not physically present and therefore is unable to move around the room to observe the physical examination. Lastly, the population of virtual assistants employed by Hello Rache seems to be restricted to specifically trained RNs in the Philippines. These virtual assistants are specially vetted for working in the medical field, and their position as a virtual assistant is their career, which provides a specialized workforce to help physicians be more effective in their work. It also shows stark contrast to the prehealth professionals that make up the majority of conventional scribes for whom scribing is a stepping stone into the medical field rather than a career path. This offers a more comprehensive approach to reducing the physician's workload but also contributes to a more detached clinical experience for the virtual assistant.

\section{Final Thoughts}

Both conventional and virtual scribing modalities provide assistance to maximize efficiency and reduce the physician's workload. ${ }^{3}$ Both methods achieve the same goal, but they have unique long-term impact on the physician, scribe, and most importantly the patient. Artificial intelligence provides an intriguing approach to minimizing work in the medical setting, but it does not have the successful history of utilization and longitudinal clinical impact on the scribe that is achieyed through traditional scribing. It is important to consider the personal and professional growth that early clinical experiences provide for scribes, especially because the majority pursue a career in the medical field. Human scribes will continue to be the status quo when opposing the increased requirements of the EMR, but the implementation of AI sparks the need for more in-depth research and comparisons. Lastly, it is essential to uncover what the patient may prefer. Conventional scribing has been successfully utilized and accepted by patients in the clinical setting for years, but investigations of the efficacy and satisfaction of virtual scribing are still needed. Although both provide an advantageous approach to maximizing the patientphysician time in the dermatology clinic, one cannot say for certain that AI will be welcomed the same way as modern-day human scribes.

\section{REFERENCES}

1. Arndt BG, Beasley JW, Watkinson MD, et al. Tethered to the EHR: primary care physician workload assessment using EHR event log data and time-motion observations [published online September 2017]. Ann Fam Med. doi:10.1370/afm.2121

2. Robert J, Piemonte N, Truten J. The reflective scribe: encouraging critical self-reflection and professional development in pre-health education. J Med Humanit. 2018;39:447-454. doi:10.1007/s10912-018-9541-1

3. Berger E. Medical scribe industry booms: rapid rise leads to questioning. Ann Emerg Med. 2015;65:A13. doi:10.1016/j.annemergmed.2015.02.016 